\title{
Zircônia tetragonal estabilizada por ítria: comportamento mecânico, adesão e longevidade clínica
}

\section{(Yttria-stabilized tetragonal zirconia: mechanical behavior, adhesion and clinical longevity)}

\author{
Y. D. Belo, Q. N. Sonza, M. Borba, A. D. Bona \\ Faculdade de Odontologia da Universidade de Passo Fundo, BR286, Bairro S. José, Passo Fundo, RS 99052-900
}

\begin{abstract}
Resumo
A zircônia tetragonal policristalina estabilizada por ítria (Y-TZP) vem sendo amplamente empregada na Odontologia como material de infraestrutura (IE) de coroas e próteses parciais fixas devido às suas características mecânicas como alta resistência e tenacidade à fratura. $\mathrm{O}$ objetivo desta revisão de literatura é buscar evidências em estudos in vitro e in vivo a respeito do comportamento mecânico, adesão e longevidade clínica de próteses fixas confeccionadas com IE de Y-TZP. Foram obtidos artigos, na base de dados online Medline/Pubmed, seguindo a seguinte combinação de palavras-chaves: zircônia tetragonal estabilizada por ítria ("Y-TZP"), adesão ("adhesion"; "bonding"), propriedades mecânicas ("mechanical properties"), estudos clínicos ("long-term clinical trials"), longevidade ("longevity"). A busca abrangeu os anos de 1990 a 2012. De acordo com a literatura, a Y-TZP apresenta propriedades mecânicas superiores às demais cerâmicas odontológicas devido a um mecanismo de tenacificação associado à transformação de fase cristalina (transformation toughening). Ainda, a silicatização associada a silanização tem sido indicada como o tratamento de superfície mais adequado para cimentação adesiva de IE em Y-TZP, além do uso de um cimento resinoso contendo monômeros fosfatados (MDP). Nos estudos clínicos, a Y-TZP tem mostrado altas taxas de sucesso como IE de coroas unitárias e PPFs. Apesar do comportamento mecânico e de união da Y-TZP não estarem completamente esclarecidos, estudos apontam resultados promissores em relação à aplicação clínica deste material.
\end{abstract}

Palavras-chave: zircônia, cerâmicas dentais.

\begin{abstract}
Yttrium stabilized tetragonal zirconia polycrystal (Y-TZP) has been widely used in Dentistry as framework material for dental crowns and fixed partial dentures (FPDs) due to its mechanical characteristics, such as high fracture strength and toughness. The objective of this literature review is to search for scientific evidence concerning the mechanical behavior, adhesion and clinical longevity of Y-TZP-based restorations. Literature was obtained from online Medline/Pubmed database using the following keywords combination: Y-TZP; adhesion; bonding; mechanical properties; long-term clinical trials; longevity. Articles from 1990 to 2012 were searched. According to the literature, Y-TZP-based ceramic shows superior mechanical properties when compared to other dental ceramics due to a crystal phase transformation toughening mechanism. In addition, silica coating associated with silanization is recommended as the most successful surface treatment for Y-TZP adhesive cementation, and the use of a MDPcontaining resinous cement system. According to the clinical studies, Y-TZP has high success rates when used as framework material for single crowns and FPDs. Although the mechanical and adhesive behavior of Y-TZP is not completely understood, studies show Y-TZP as a promising ceramic for clinical application.
\end{abstract}

Keywords: zirconia, dental ceramics.

\section{INTRODUÇÃO}

O uso das cerâmicas como material de substituição da estrutura dentária está baseado em características favoráveis como alta biocompatibilidade, altos valores de dureza, inércia química e suas características estéticas [1]. Estruturalmente, estes materiais podem ser divididos em vidros amorfos, cerâmicas parcialmente cristalinas (com alto ou baixo teor de vidro) e cerâmicas policristalinas (sem conteúdo vítreo) [1-3].

As cerâmicas policristalinas, como a zircônia tetragonal estabilizada com ítria (Y-TZP), podem ser utilizadas como componentes para implantes dentários, como infraestrutura para coroas unitárias e próteses parciais fixas totalmente cerâmicas na região anterior e posterior, como braquetes ortôdonticos e pinos intraradiculares [1]. O uso da Y-TZP como material de infraestrutura para próteses fixas totalmente cerâmicas foi impulsionado pela introdução da tecnologia CAD-CAM (computer aided design - computer aided machining) na Odontologia. Essa cerâmica apresenta um comportamento mecânico superior ao observado pelas demais cerâmicas odontológicas, sendo atualmente o material de escolha para a confecção de restaurações submetidas à alta concentração de tensões. Além das diferenças estruturais, a Y-TZP apresenta algumas características adesivas e mecânicas diferentes dos vidros amorfos e das 
cerâmicas parcialmente cristalinas [1,2]. Desta forma, os objetivos da presente revisão de literatura são descrever as características mecânicas e adesivas da Y-TZP e apresentar dados de longevidade das próteses totalmente cerâmicas confeccionadas com este material.

\section{MÉTODOS}

A estratégia de pesquisa envolveu uma busca de artigos, na língua inglesa, a partir da base de dados online Medline/ Pubmed, utilizando a seguinte combinação de palavraschaves: zircônia tetragonal estabilizada por ítria ("Y-TZP"), "AND” adesão ("adhesion"; "bonding"), "OR" propriedades mecânicas ("mechanical properties"), "OR" estudos clínicos ("long-term clinical trials"), "OR" longevidade ("longevity"). A busca abrangeu os anos de 1990 a 2012. Alguns artigos anteriores ao ano de 1990 foram incluídos devido a sua relevância para a pesquisa.

\section{REVISÃO DE LITERATURA}

Devido à grande quantidade de informações obtidas, a presente revisão de literatura foi dividida em tópicos: (1) comportamento mecânico; (2) adesão; (3) longevidade clínica.

\section{Comportamento mecânico}

As cerâmicas policristalinas à base de zircônia podem ser classificadas em três tipos de acordo com a sua microestrutura: FSZ(fully stabilized zirconia), PSZ (partially stabilized zirconia) e TZP (tetragonal zirconia polycristals). Na FSZ, a zircônia se encontra em sua forma cúbica, sendo geralmente obtida adicionando-se grandes quantidades de estabilizadores (mais de $8 \mathrm{~mol} \%$ de óxido de ítrio). A PSZ é constituída de partículas nanométricas nas formas monoclínica ou tetragonal que são precipitadas na matriz de zircônia cúbica. Esses materiais são geralmente obtidos por meio de adição de óxido de cálcio ou magnésio. ATZPé o tipo de cerâmica à base de zircônia mais utilizado para aplicação odontológica e é constituída predominantemente pela fase tetragonal, embora possa conter pequenas quantidades de fase cúbica secundária, e geralmente é estabilizada com óxido de ítrio (3\%-6\% em peso), dando origem à Y-TZP (zircônia tetragonal estabilizada com ítria) [4].

O comportamento mecânico dos materiais à base de zircônia está associado com um aumento de tenacidade por transformação de fase (transformation toughening). A zircônia pode assumir três formas cristalográficas dependendo da temperatura. A estrutura é monoclínica desde a temperatura ambiente até $1170^{\circ} \mathrm{C}$, tetragonal entre $1170^{\circ} \mathrm{C}$ e $2370^{\circ} \mathrm{C}$ e cúbica acima de $2370^{\circ} \mathrm{C}$ até o ponto de fusão $[4,5]$. Com o objetivo de estabilizar a fase tetragonal à temperatura ambiente são adicionados óxidos a zircônia pura como os óxidos de cálcio $(\mathrm{CaO})$, de magnésio $(\mathrm{MgO})$, de ítrio $\left(\mathrm{Y}_{2} \mathrm{O}_{3}\right)$ ou de cério $\left(\mathrm{CeO}_{2}\right)$. Quando este material estabilizado é submetido a tensões (ex: mastigação, desgaste e polimento), ocorre uma transformação da fase cristalina tetragonal para monoclínica. Como a forma monoclínica ocupa um volume de 3 a $5 \%$ maior do que os grãos tetragonais, o resultado final é geração de tensões de compressão e nucleação de micro-trincas (microcrack toughening) ao redor do defeito, impedindo que a trinca se propague e leve à fratura do material. Esse mecanismo é o principal responsável pelo fato da Y-TZP ser a cerâmica odontológica que apresenta as melhores propriedades mecânicas [6].

A confecção de próteses dentárias utilizando a cerâmica Y-YZP foi impulsionada pela introdução da tecnologia CAD-CAM na Odontologia. Esta cerâmica é indicada como material de infraestrutura de próteses fixas porque apresenta alta resistência à fratura e opacidade, devido a seu alto conteúdo cristalino, e deve ser recoberta com uma cerâmica vítrea translúcida para produzir uma restauração com cor e forma semelhante à estrutura dental. Para a confecção de infraestruturas de Y-TZP existem blocos parcialmente sinterizados, que são submetidos a um processo de sinterização final após a usinagem da infraestrutura, e blocos densamente sinterizados por meio de prensagem isostática a quente (hot isostatic pressing - HIP). Quando os blocos parcialmente sinterizados são utilizados, a infraestrutura é usinada com um tamanho, aproximadamente, $20 \%$ maior do que o tamanho final desejado, para compensar a contração de sinterização. A usinagem de blocos densamente sinterizados pode produzir restaurações com melhor adaptação, porém esse processo é demorado e envolve grande desgaste dos instrumentos. Já o uso de blocos parcialmente sinterizados aumenta a eficiência do processo de usinagem [7].

Para aplicação odontológica, a microestrutura da cerâmica Y-TZP é composta por grãos eqüiaxiais com diâmetro entre 0,2 a $0,5 \mu \mathrm{m}$, dependendo da temperatura de sinterização $[8,9]$. Esse sistema cerâmico apresenta valores médios de resistência à flexão variando entre 700 e $1500 \mathrm{MPa}$ e tenacidade à fratura entre 4,4 e 9,4 MPa.m ${ }^{1 / 2}[8,10-18]$. Foram reportados para Y-TZP valores de dureza de $\sim 12 \mathrm{GPa}$ e módulo de elasticidade entre 220 e $240 \mathrm{GPa}[8,10]$. Foi relatado um valor alto de módulo de Weibull para a Y-TZP $(18,4)$ [11, 12]. Entretanto, outros estudos encontraram valores mais baixos de módulo de Weibull, variando entre 10 e 16 [13-15, 17, 19]. Apesar da Y-TZP apresentar um mecanismo de tenacificação que eleva suas propriedades mecânicas, este mesmo mecanismo pode resultar em um comportamento indesejável: a degradação das propriedades mecânicas em baixa temperatura (low temperature degradation- LTD) [9, 20]. Foi observado em um estudo que os grãos da Y-TZP podem apresentar uma transformação lenta da fase tetragonal para monoclínica nos grãos de zircônia da superfície, em ambiente úmido e temperaturas relativamente baixas $\left(150-400{ }^{\circ} \mathrm{C}\right)$. A transformação ocorre através de um processo de nucleação e crescimento dos grãos e é acompanhada pelo aumento de volume, provocando tensões e micro-trincas nos grãos vizinhos. Esse processo de degradação da superfície é exacerbado pela presença de água. $\mathrm{O}$ crescimento da zona de transformação resulta em aumento do número de micro-trincas, extrusão dos grãos e 
aumento da rugosidade superficial, o que leva à degradação da resistência. Qualquer fator que afete a estabilidade da zircônia tetragonal pode afetar esse processo de degradação $[4,9]$. Entre esses fatores estão o tamanho médio do grão $[22,23]$, a quantidade de estabilizador [5] e a presença de tensões residuais [15, 24-26]. Grãos de Y-TZP acima de um tamanho crítico são menos estáveis e mais susceptíveis à degradação, enquanto que os de tamanho menor $(<1 \mu \mathrm{m})$ apresentam menor degradação [22]. Entretanto, tamanhos muito reduzidos $(<0,2 \mu \mathrm{m})$ restringem o aumento da tenacidade por transformação levando a uma redução das propriedades mecânicas [23]. O processo de sinterização interfere diretamente no tamanho de grão, sendo que altas temperaturas e longo tempo de sinterização resultam em maior tamanho de grão [9]. A diminuição na concentração de estabilizador pode resultar em menor estabilidade dos grãos tetragonais, gerando zonas de nucleação para transformação espontânea da fase tetragonal para monoclínica [5, 9].

Tensões residuais podem ser introduzidas durante o processamento das restaurações. Estudos investigaram o efeito da usinagem, alterações térmicas, e dos procedimentos de acabamento e polimento no comportamento mecânico da cerâmica Y-TZP e encontraram resultados controversos $[15,25,26]$. Os procedimentos de usinagem e acabamento podem induzir uma camada de tensões de compressão na superfície da cerâmica melhorando as propriedades mecânicas do material. No entanto, também podem produzir defeitos profundos que ultrapassam essa camada de compressão, aumentado a susceptibilidade à degradação em baixas temperaturas $[15,25,26]$. O tratamento térmico da Y-TZP com temperaturas entre 900 e $1000^{\circ} \mathrm{C}$ pode induzir a transformação reversa da fase monoclínica para tetragonal. Por isso, sugere-se que a sinterização da camada de porcelana sobre a infraestrutura da Y-TZP possa provocar essa reversão de fase, resultando na liberação das tensões de compressão presentes na superfície cerâmica e, conseqüentemente, na diminuição da resistência à fratura [16].

\section{Adesão}

Como relatado acima, o aumento do tamanho médio de grão na microestrutura das cerâmicas tem uma relação direta com um aumento nos valores de propriedades mecânicas, como resistência e tenacidade à fratura. Porém, este aumento e, consequentemente, redução do conteúdo vítreo, tornaram as cerâmicas policristalinas resistentes ao condicionamento ácido, ou seja, o condicionamento ácido não produz alterações topográficas suficientes para proporcionar uma união adesiva adequada $[10,27,28]$. Assim, com o objetivo de alcançar uma união adesiva consistente entre cerâmicas ácido-resistentes, como a Y-TZP, e materiais resinosos, alguns tratamentos de superfície são propostos para as superfícies cerâmicas. Entre os tratamentos mais utilizados estão o jateamento de superfície com partículas de alumina [15, 29,30], a silanização [31,32], a silicatização [27, 30,33] e o uso de um monômero fosfatado (10-methacryloxydecyl dihydrogen phosphate - MDP) que pode estar presente no cimento resinoso ou ainda associado ao agente de união silano $[34,35]$. O jateamento com partículas de alumina tem por objetivo limpar, aumentar a rugosidade superficial e ativar a superfície jateada [15]. Este procedimento é motivo de discussão entre autores, pois alguns estudos atribuem características positivas a este procedimento, determinando que o jateamento iniciaria uma transformação de fase na zircônia resultando na formação de tensão de compressão na superfície do material, restringindo a propagação da fratura na ponta da trinca $[15,29]$, enquanto outros afirmam que o impacto das partículas jateadas pode criar defeitos na superfície cerâmica gerando áreas de concentração de tensão que pode conduzir à falha do material $[36,37]$. Um estudo demonstrou que o polimento, o jateamento e os tratamentos para LTD não degradaram significativamente a resistência da Y-TZP; além disso, o jateamento aumentou a resistência á flexão [38]. Porém, os resultados indicaram menor confiabilidade para o grupo jateado em relação ao desempenho clínico. Foi sugerido que em condições clínicas o jateamento pode desenvolver áreas de tensão na superfície cerâmica diminuindo a resistência ao longo do tempo. Por outro lado, outra investigação reportou que o jateamento com pressões relativamente baixas associadas a primers adesivos podem ser empregadas para minimizar possíveis danos à superfície cerâmica [39].

Os agentes de união silano são compostos que possuem na sua composição básica átomos de silício, além de dois grupamentos ativos: um organofuncional (ex. vinyl, epoxy e amino) que permite a união com a matriz orgânica e o outro é um hidrolisável (ex. methoxy, ethoxy), que reage com a matriz inorgânica como, por exemplo, a sílica $[1,40]$. Na união às cerâmicas, os silanos formam grupos silanóis que por sua vez se unem, por meio de reação de condensação, à sílica presente na superfície cerâmica, formando uma rede siloxano [31, 32, 41]. Porém, a aplicação de silano somente, na superfície de cerâmicas com alto conteúdo cristalino e policristalinas, tem demonstrado valores considerados baixos de adesão, já que a quantidade de sílica disponível para a reação com o silano, nesse tipo de material, é baixa ou praticamente inexistente [31, 42]. Apesar disso, estudos demonstram que a associação com a silicatização produz valores considerados aceitáveis de tal propriedade [27, 30, 33, 43]. A silicatização é um método de deposição de sílica na superfície de substratos como metais, cerâmicas e compósitos por meio de uma ação triboquímica, ou seja, uma união química obtida através da utilização de energia cinética. Esta união ocorre por jateamento destas partículas modificadas por sílica, que produzem uma alteração na topografia da superfície, além da formação de uma camada de sílica que pode penetrar até $15 \mu \mathrm{m}$ no substrato. Uma vez formada esta camada de sílica topograficamente irregular, os agentes de união silano podem ser aplicados de modo a formar uma união química entre cerâmica e o material resinoso [30, $40,44]$. Outra opção de tratamento de superfície é o uso de agentes que contenham um monômero fosfatado como o MDP, que possui a capacidade de se unir quimicamente com óxidos metálicos [34]. Esta característica permite que o MDP 
seja utilizado para o tratamento de superfícies cerâmicas que apresentam, por exemplo, zircônia na composição, visto que o zircônio é um elemento químico da família dos metais [1]. Desta forma, o MDP age sobre os óxidos metálicos na superfície, promovendo união entre o material resinoso e a estrutura cerâmica [35]. Estudos demonstram que este componente promove uma união adequada em condições secas, porém, é instável hidrolítica e termicamente. Desta forma, tem sido proposta a aplicação deste componente em associação com agentes de união silanos, com o objetivo de obter bons resultados de adesão em condições secas e de envelhecimento em ambiente úmido. Além do MDP, existem outros monômeros que apresentam afinidade por óxidos metálicos e que podem estar presentes nos agentes de união silanos como o VBATDT (6-(4-vinylbenzyl-n-propyl) amino-1,3,5-triazine-2,4-dithione), MEPS (thiophosphoric methacrylate) e MTU-6 (6-methacryloyloxyhexyl-2thiouracil-5-carboxylate) $[35,45]$.

Portanto, o tratamento de superfície das cerâmicas policristalinas não está totalmente estabelecido e gera controvérsias entre pesquisadores e clínicos; apesar disso, a silicatização associada à silanização parece demonstrar bons resultados de união adesiva para estas cerâmicas [43, 46, 47]. Porém, a dúvida maior está em torno da estabilidade de união por longos períodos, pois, alguns estudos determinam esta união como sendo estável [45, 48], enquanto outros demonstram instabilidade na interface adesiva devido ao desprendimento das partículas de sílica da superfície cerâmica $[42,49]$. Um estudo recente avaliou a resistência de união da cerâmica Y-TZP com diferentes tratamentos de superfície. Foram utilizados a silicatização, silano contendo MDP, jateamento com alumina e um primer metálico. Após termociclagem e estocagem em água por 60 dias os resultados demonstraram que a silicatização promoveu os melhores resultados de união [50].

O sucesso clínico de restaurações cerâmicas está fortemente associado ao preparo da estrutura dentária e ao procedimento de cimentação. Existem vários tipos de cimentos que podem ser utilizados para a cimentação de estruturas cerâmicas tais como cimentos de ionômero de vidro convencionais, ionômero de vidro modificado por resina, cimento de fosfato de zinco e cimentos resinosos convencionais e auto-adesivos [46]. Desta forma, a cimentação pode ser realizada de forma adesiva através do uso de sistemas adesivos ou de forma não-adesiva com a utilização de cimentos que atuam somente através do embricamento mecânico como é o caso do cimento de fosfato de zinco [1,51]. Os cimentos resinosos normalmente utilizados apresentam na sua composição monômeros como bis-GMA (bisphenol glycydil methacrylate), 4-META com metil metacrilato (4-META/MMA), UDMA (urethane dimethacrylate), TEGMA (triethyleneglycol dimethacrylate) ou MDP (10-methacryloxydecyl dihydrogen phosphate), associados a partículas inorgânicas que diminuem o coeficiente de expansão térmica e a contração de polimerização [43, 52]. De acordo com a polimerização, estes podem ser divididos em cimentos quimicamente ativados, fotopolimerizáveis e duais. Os cimentos resinosos são frequentemente utilizados para a cimentação de restaurações cerâmicas por proporcionarem um selamento marginal e um aumento na resistência à fratura quando comparados com a cimentação não-adesiva [53].

Uma investigação demonstrou que o cimento de fosfato de zinco, ionômero de vidro convencional e ionômero de vidro modificado por resina não apresentaram união estável com a zircônia, sendo que os melhores resultados de união foram alcançados com um cimento resinoso auto-adesivo [46]. Outros estudos com diferentes metodologias também avaliaram a união entre as cerâmicas com alto conteúdo cristalino cimentadas com materiais resinosos com e sem a presença de monômeros fosfatados [34, 35]. Outros monômeros com afinidade por óxidos metálicos também podem estar presentes nos cimentos resinosos como o grupo anidrido presente no monômero 4-META e o metacrilato ester fosfórico [34, 35, 54].

\section{Longevidade clínica}

A longevidade de restaurações cerâmicas é variável e dependente de fatores relevantes como o tipo de material cerâmico, o processo de fabricação, a espessura do conector, bem como das condições do preparo da estrutura dentária e da cimentação da peça protética $[46,55,56]$. Estudos que avaliaram a longevidade da Y-TZP têm demonstrado resultados clínicos interessantes.

Com relação à extensão da prótese, coroas unitárias apresentam resultados clínicos mais satisfatórios do que PPFs. Um estudo que acompanhou 18 PPFs e 50 coroas unitárias com infraestrutura de Y-TZP por um período de 3 anos observou 8 falhas de caráter técnico ou biológico, sendo que todas ocorreram nas PPFs [57].

Embora não existam muitos resultados disponíveis com coroas unitárias, estudos demonstram longevidade significativa de estruturas de Y-TZP. Coroas com infraestrutura em zircônia em dentes anteriores severamente destruídos foram avaliadas e após um período de acompanhamento de 3 anos um índice de sobrevida de $100 \%$ foi verificado [58]. Outro estudo avaliou 102 coroas em dentes anteriores e posteriores. Em um período médio de avaliação de 20,9 meses os autores não verificaram nenhuma fratura em infraestrutura [59]. Da mesma forma, outra investigação clínica não relatou nenhuma fratura da infraestrutura a base de zircônia nem cárie secundária em um período 3 anos [60].

Em relação as PPFs, estudos com acompanhamento de 3 anos demonstraram índices consideráveis de sobrevida de estruturas de zircônia. Trinta e quatro próteses parciais fixas foram acompanhadas e verificou-se que todas as infraestruturas de Y-TZP permaneceram intactas, havendo apenas lascamento da cerâmica de cobertura em dois casos [61]. Resultados semelhantes foram encontrados [62, 63] verificando um índice de sobrevida de $90 \%$ para as PPFs (infraestrutura e cerâmica de cobertura) e de $95,2 \%$ para as infraestruturas de zircônia. Em um acompanhamento de 5 
anos verificou-se um índice de sobrevida de $74 \%$ quando a cerâmica Y-TZP foi utilizada como infraestrutura de próteses com 3 elementos. Os autores observaram como responsáveis pelas falhas fatores como a cárie secundária $(21,7 \%)$ e o lascamento da porcelana (15,3\%) [64]. Outro estudo com acompanhamento mais longo, de 10 anos, reportou um índice de sucesso de $91,5 \%$ para as infraestruturas a base de zircônia, observando apenas 3 fraturas. Por outro lado, o índice de sobrevida das PPFs foi de $67 \%$. Segundo os autores, a fratura da cerâmica de cobertura, deficiência marginal e cárie secundária foram às falhas verificadas [65]. Uma revisão de literatura avaliando a desempenho clínico de PPFs com infraestrutura de zircônia verificou um índice de sobrevida de $73,9 \%$ - 100\% entre 2 - 5 anos de avaliação estando entre as principais causas de falha a fratura da cobertura de porcelana, fratura da infraestrutura e cárie secundária [66].

A fratura da infraestrutura de Y-TZP é difícil de ocorrer, sendo mais comum nas PPFs [67]; porém, a fratura da cerâmica de cobertura é um problema comum, sendo que os índices reportados podem chegar a $50 \%$ em avaliações de 1 a 2 anos enquanto que em restaurações metalocerâmicas os índices são consideravelmente inferiores [9, $56,58]$. Um estudo clínico que avaliou restaurações metalocerâmicas e totalmente cerâmicas, em 3 anos, demonstrou um índice de sobrevida de $100 \%$ para os dois grupos, porém, foram relatados $25 \%$ de pequenas fraturas na cerâmica de cobertura de restaurações totalmente cerâmicas e 19\% nas metalo-cerâmicas [69]. Alguns motivos são apontados como possíveis responsáveis por essas falhas nas restaurações totalmente cerâmicas, como problemas na união e na compatibilidade térmica entre infraestrutura e cerâmica de cobertura, falta de um suporte uniforme para a cerâmica de cobertura e redução dos dopantes estabilizadores (ex: ítria) que podem resultar em alterações nas fases da zircônia ou em expansão térmica local anisotrópica [1, 69, 70]. Em relação à extensão das PPFs, um estudo verificou que próteses de 4 e 5 elementos apresentaram uma probabilidade 4,9 vezes maior para o lascamento da cerâmica de cobertura do que próteses de 3 elementos [65].

\section{CONSIDERAÇÕES FINAIS}

A Y-TZP apresenta propriedades mecânicas superiores às demais cerâmicas odontológicas devido a um mecanismo de tenacificação associado à transformação de fase cristalina (transformation toughening). Entretanto, este mesmo mecanismo pode resultar na degradação das propriedades mecânicas em baixa temperatura (low temperature degradation- LTD). Fatores como o tamanho médio do grão, a quantidade de estabilizador e as tensões residuais podem estar envolvidos com esse mecanismo de degradação. Porém, não existe um consenso de como esses fatores afetam as propriedades mecânicas da Y-TZP. A Y-TZP é uma cerâmica ácido-resistente, o que influencia diretamente o protocolo de cimentação adesiva de restaurações com essa cerâmica na infraestrutura. Alguns tratamentos de superfície foram propostos para melhorar a união com o cimento resinoso, mas ainda existem controvérsias entre pesquisadores e clínicos. Apesar disso, a silicatização associada à silanização parece mostrar bons resultados de união adesiva para estas cerâmicas. Além disso, é recomendado o uso de um cimento resinoso à base de MDP. Baseado nos estudos clínicos analisados, a cerâmica Y-TZP parece ser adequada para a fabricação de infraestruturas de coroas unitárias e PPFs. A quantidade de fraturas do material de infraestrutura não é significativa, sendo que os modos de falha mais observados foram cárie secundária e lascamento da camada de porcelana. Apesar disso, estudos clínicos com maior tempo de acompanhamento são necessários para compreender melhor o comportamento de cerâmicas à base de zircônia.

\section{REFERÊNCIAS}

[1] A. Della Bona, "Bonding to ceramics: scientific evidences for clinical dentistry", $1^{a}$ Ed., Artes Médicas, S. Paulo, SP (2009) 254.

[2] J. R. Kelly, P. Benetti, Ceramic materials in dentistry: historical evolution and current practice, Aust. Dent. J. 56 Suppl 1 (2011) 84.

[3] J. B. Quinn, V. Sundar, I. K. Lloyd, Influence of microstructure and chemistry on the fracture toughness of dental ceramics, Dent. Mater. 19 (2003) 603.

[4] J. Chevalier, L. Gremillard, The tetragonal-monoclinic transformation in zirconia: lessons learned and future trends, J. Am. Ceram. Soc. 92 (2009) 1901.

[5] R. H. J. Hannink, P. M. Kelly, B. C. Muddle, Transformation toughening in zirconia-containing ceramics, J. Am. Ceram. Soc. 83 (2000) 461.

[6] J. R. Kelly, I. Denry, Stabilized zirconia as a structural ceramic: An overview, Dent. Mater. 24 (2008) 289.

[7] J. Tinschert, G. Natt, S. Hassenpflug, H. Spiekermann, Status of current CAD/CAM technology in dental medicine, Int. J. Comput. Dent. 7 (2004) 25.

[8] M. Borba, M. D. de Araujo, K. A. Fukushima, H. N. Yoshimura, P. F. Cesar, J. A. Griggs, A. Della Bona, Effect of the microstructure on the lifetime of dental ceramics, Dent. Mater. 27 (2011) 710.

[9] I. Denry, J. R. Kelly, State of the art of zirconia for dental applications, Dent. Mater. 24 (2008) 299.

[10] M. Guazzato, M. Albakry, S. P. Ringer, M. V. Swain, Strength, fracture toughness and microstructure of a selection of all-ceramic materials. Part II. Zirconia-based dental ceramics, Dent. Mater. 20 (2004) 449.

[11] J. Tinschert, D. Zwez, R. Marx, K. J. Anusavice, Structural reliability of alumina-, feldspar-, leucite-, micaand zirconia-based ceramics, J. Dent. 28 (2000) 529.

[12] J. Tinschert, G. Natt, H. Spiekermann, K. A. Schulze, Lifetime of Alumina and Zirconia Ceramics Used for Crown and Bridge Restorations, J. Biomed. Mater. Res. Part B: Appl. Biomater. 80B (2007) 317.

[13] H. Yilmaz, C. Aydin, B. E. Gul, Flexural strength and fracture toughness of dental core ceramics, J. Prosthet. Dent. 98 (2007) 120. 
[14] R. G. Luthardt, M. Holzhuter, O. Sandkuhl, V. Herold, J. D. Schnapp, E. Kuhlisch, M. Walter, Reliability and properties of ground Y-TZP-zirconia ceramics, J. Dent. Res. 81 (2002) 487.

[15] T. Kosmac, C. Oblak, P. Jevnikar, N. Funduk, L. Marion, The effect of surface grinding and sandblasting on flexural strength and reliability of Y-TZP zirconia ceramic, Dent. Mater. 15 (1999) 426.

[16] M. Oilo, N. R. Gjerdet, H. M. Tvinnereim, The firing procedure influences properties of a zirconia core ceramic, Dent. Mater. 24 (2008) 471.

[17] S. Liu, I. Chen, Fatigue of yttria-stabilized zirconia: I, fatigue damage, fracture origins, and lifetime prediction, J. Am. Ceram. Soc. 74 (1991) 1197.

[18] B. Taskonak, J. Yan, J. J. Mecholsky, Jr., A. Sertgoz, A. Kocak, Fractographic analyses of zirconia-based fixed partial dentures, Dent. Mater. 24 (2008) 1077.

[19] E. C. Teixeira, J. R. Piascik, B. R. Stoner, J. Y. Thompson, Dynamic fatigue and strength characterization of three ceramic materials, J. Mater. Sci.: Mater. Med. 18 (2007) 1219.

[20] C. Piconi, G. Maccauro, Zirconia as a ceramic biomaterial, Biomater. 20 (1999) 1.

[21] K. Kobayashi, H. Kuwajima, T. Masaki, Phase change and mechanical properties of $\mathrm{ZrO}_{2}-\mathrm{Y}_{2} \mathrm{O}_{3}$ solid electrolyte after aging, Solid State Ionics 4 (1981) 489.

[22] A. H. Heuer, N. Claussen, W. M. Kriven, M. Ruhle, Stability of tetragonal $\mathrm{ZrO}_{2}$ particles in ceramic matrices, J. Am. Ceram. Soc. 65 (1982) 642.

[23] B. A. Cottom, M. J. Mayo, Fracture toughness of nanocrystalline $\mathrm{ZrO}_{2}-3$ mol\% $\mathrm{Y}_{2} \mathrm{O}_{3}$ determined by Vickers indentation, Scripta Mater. 34 (1996) 809.

[24] S. Deville, J. Chevalier, L. Gremillard, Influence of surface finish and residual stresses on the ageing sensitivity of biomedical grade zirconia, Biomater. 27 (2006) 2186.

[25] M. Guazzato, L. Quach, M. Albakry, M. V. Swain, Influence of surface and heat treatments on the flexural strength of Y-TZP dental ceramic, J. Dent. 33 (2005) 9.

[26] J. W. Kim, N. S. Covel, P. C. Guess, E. D. Rekow, Y. Zhang, Concerns of hydrothermal degradation in CAD/ CAM zirconia, J. Dent. Res. 89 (2010) 91.

[27] A. Della Bona, M. Borba, P. Benetti, D. Cecchetti, Effect of surface treatments on the bond strength of a zirconiareinforced ceramic to composite resin, Braz. Oral Res. 21 (2007) 10.

[28] A. Della Bona, K. J. Anusavice, Microstructure, composition, and etching topography of dental ceramics, Int. J. Prosthodont. 15 (2002) 159.

[29] A. R. Curtis, A. J. Wright, G. J. Fleming, The influence of surface modification techniques on the performance of a Y-TZP dental ceramic, J. Dent. 34 (2006) 195.

[30] A. Della Bona, T. A. Donassollo, F. F. Demarco, A. A. Barrett, J. J. Mecholsky, Jr., Characterization and surface treatment effects on topography of a glass-infiltrated alumina/zirconia-reinforced ceramic, Dent. Mater. 23 (2007) 769.

[31] J. Y. Thompson, B. R. Stoner, J. R. Piascik, R. Smith,
Adhesion/cementation to zirconia and other non-silicate ceramics: where are we now?, Dent. Mater. 27 (2011) 71.

[32] J. P. Matinlinna, L. V. Lassila, M. Ozcan, A. Yli-Urpo, P. K. Vallittu, An introduction to silanes and their clinical applications in dentistry, Int. J. Prosthodont. 17 (2004) 155. [33] L. F. Valandro, M. Ozcan, M. C. Bottino, M. A. Bottino, R. Scotti, A. D. Bona, Bond strength of a resin cement to high-alumina and zirconia-reinforced ceramics: the effect of surface conditioning, J. Adhes. Dent. 8 (2006) 175.

[34] M. Kern, S. M. Wegner, Bonding to zirconia ceramic: adhesion methods and their durability, Dent. Mater. 14 (1998) 64.

[35] K. Yoshida, Y. Tsuo, M. Atsuta, Bonding of dual-cured resin cement to zirconia ceramic using phosphate acid ester monomer and zirconate coupler, J. Biomed. Mater. Res. B: Appl. Biomater. 77 (2006) 28.

[36] Y. Zhang, B. R. Lawn, K. A. Malament, P. Van Thompson, E. D. Rekow, Damage accumulation and fatigue life of particle-abraded ceramics, Int. J. Prosthodont. 19 (2006) 442.

[37] M. Uo, G. Sjogren, A. Sundh, M. Goto, F. Watari, M. Bergman, Effect of surface condition of dental zirconia ceramic (Denzir) on bonding, Dent. Mater. J. 25 (2006) 626. [38] H. P. Papanagiotou, S. M. Morgano, R. A. Giordano, R. Pober, In vitro evaluation of low-temperature aging effects and finishing procedures on the flexural strength and structural stability of Y-TZP dental ceramics, J. Prosthet. Dent. 96 (2006) 154.

[39] M. Kern, A. Barloi, B. Yang, Surface conditioning influences zirconia ceramic bonding, J. Dent. Res. 88 (2009) 817.

[40] J. P. Matinlinna, L. V. Lassila, P. K. Vallittu, Pilot evaluation of resin composite cement adhesion to zirconia using a novel silane system, Acta Odontol. Scand. 65 (2007) 44.

[41] A. Della Bona, C. Shen, K. J. Anusavice, Work of adhesion of resin on treated lithia disilicate-based ceramic, Dent. Mater. 20 (2004) 338.

[42] L. Chen, B. I. Suh, J. Kim, F. R. Tay, Evaluation of silica-coating techniques for zirconia bonding, Am. J. Dent. 24 (2011) 79.

[43] M. Ozcan, C. Cura, L. F. Valandro, Early bond strength of two resin cements to Y-TZP ceramic using MPS or MPS/4META silanes, Odontology 99 (2011) 62.

[44] T. T. Heikkinen, L. V. Lassila, J. P. Matinlinna, P. K. Vallittu, Effect of operating air pressure on tribochemical silica-coating, Acta Odontol. Scand. 65 (2007) 241.

[45] R. Tanaka, A. Fujishima, Y. Shibata, A. Manabe, T. Miyazaki, Cooperation of phosphate monomer and silica modification on zirconia, J. Dent. Res. 87 (2008) 666.

[46] A. Piwowarczyk, H. C. Lauer, J. A. Sorensen, The shear bond strength between luting cements and zirconia ceramics after two pre-treatments, Oper. Dent. 30 (2005) 382.

[47] H. L. de Castro, P. H. Corazza, A. Paes-Junior Tde, A. Della Bona, Influence of Y-TZP ceramic treatment and different resin cements on bond strength to dentin, Dent. Mater. 28 (2012) 1191. 
[48] M. B. Blatz, G. Chiche, S. Holst, A. Sadan, Influence of surface treatment and simulated aging on bond strengths of luting agents to zirconia, Quintessence Int. 38 (2007) 745.

[49] D. M. Qeblawi, C. A. Munoz, J. D. Brewer, E. A. Monaco, Jr., The effect of zirconia surface treatment on flexural strength and shear bond strength to a resin cement, J. Prosthet. Dent. 103 (2010) 210.

[50] P. Cristoforides, R. Amaral, L. G. May, M. A. Bottino, L. F. Valandro, Composite resin to yttria stabilized tetragonal zirconia polycrystal bonding: comparison of repair methods, Oper. Dent. 37 (2012) 263.

[51] M. B. Blatz, Adhesive cementation of high-strength ceramics, J. Esthet. Restor. Dent. 19 (2007) 238.

[52] I. B. Lee, C. M. Um, Thermal analysis on the cure speed of dual cured resin cements under porcelain inlays, J. Oral Rehabil. 28 (2001) 186.

[53] M. B. Blatz, S. Oppes, G. Chiche, S. Holst, A. Sadan, Influence of cementation technique on fracture strength and leakage of alumina all-ceramic crowns after cyclic loading, Quintessence Int. 39 (2008) 23.

[54] M. Ozcan, S. Kerkdijk, L. F. Valandro, Comparison of resin cement adhesion to Y-TZP ceramic following manufacturers' instructions of the cements only, Clin. Oral Investig. 12 (2008) 279.

[55] A. Della Bona, J. R. Kelly, The clinical success of allceramic restorations, J. Am. Dent. Assoc. 139 Suppl (2008) $8 \mathrm{~S}$.

[56] V. C. Bachhav, M. A. Aras, Zirconia-based fixed partial dentures: a clinical review, Quintessence Int. 42 (2011) 173. [57] F. Beuer, M. Stimmelmayr, W. Gernet, D. Edelhoff, J. F. Guh, M. Naumann, Prospective study of zirconia-based restorations: 3-year clinical results, Quintessence Int. 41 (2010) 631.

[58] J. Schmitt, M. Wichmann, S. Holst, S. Reich, Restoring severely compromised anterior teeth with zirconia crowns and feather-edged margin preparations: a 3-year follow-up of a prospective clinical trial, Int. J. Prosthodont. 23 (2010) 107.

[59] C. E. Poggio, R. Dosoli, C. Ercoli, A retrospective analysis of 102 zirconia single crowns with knife-edge margins, J. Prosthet. Dent. 107 (2012) 316.
[60] A. Ortorp, M. L. Kihl, G. E. Carlsson, A 3-year retrospective and clinical follow-up study of zirconia single crowns performed in a private practice, J. Dent. 37 (2009) 731.

[61] R. J. Crisp, A. J. Cowan, J. Lamb, O. Thompson, N. Tulloch, F. J. Burke, A clinical evaluation of all-ceramic bridges placed in patients attending UK general dental practices: three-year results, Dent. Mater. 28 (2012) 229.

[62] J. Pelaez, P. G. Cogolludo, B. Serrano, J. F. Lozano, M. J. Suarez, A prospective evaluation of zirconia posterior fixed dental prostheses: three-year clinical results, J. Prosthet. Dent. 107 (2012) 373.

[63] F. Beuer, D. Edelhoff, W. Gernet, J. A. Sorensen, Three-year clinical prospective evaluation of zirconiabased posterior fixed dental prostheses (FDPs), Clin. Oral Investig. 13 (2009) 445.

[64] I. Sailer, A. Feher, F. Filser, L. J. Gauckler, H. Luthy, C. H. Hammerle, Five-year clinical results of zirconia frameworks for posterior fixed partial dentures, Int. J. Prosthodont. 20 (2007) 383.

[65] C. Sax, C. H. Hammerle, I. Sailer, 10-year clinical outcomes of fixed dental prostheses with zirconia frameworks, Int. J. Comput. Dent. 14 (2011) 183.

[66] P. Triwatana, N. Nagaviroj, C. Tulapornchai, Clinical performance and failures of zirconia-based fixed partial dentures: a review literature, J. Adv. Prosthodont. 4 (2012) 76.

[67] B. Al-Amleh, K. Lyons, M. Swain, Clinical trials in zirconia: a systematic review, J. Oral Rehabil. 37 (2010) 641.

[68] C. Larsson, P. Vult von Steyern, B. Sunzel, K. Nilner, Allceramic two-to five-unit implant-supported reconstructions. A randomized, prospective clinical trial, Swed. Dent. J. 30 (2006) 45.

[69] I. Sailer, J. Gottnerb, S. Kanelb, C. H. Hammerle, Randomized controlled clinical trial of zirconia-ceramic and metal-ceramic posterior fixed dental prostheses: a 3-year follow-up, Int. J. Prosthodont. 22 (2009) 553.

[70] D.-J. Kim, Effect of $\mathrm{Ta}_{2} \mathrm{O}_{5}, \mathrm{Nb}_{2} \mathrm{O}_{5}$, and $\mathrm{HfO}_{2}$ alloying on the transformability of $\mathrm{Y}_{2} \mathrm{O}_{3-}$ stabilized tetragonal $\mathrm{ZrO}_{2}$, J. Am. Ceram. Soc. 73 (1990) 115-20.

(Rec. 27/12/2012, Ac. 20/05/2013) 